\title{
Psychological Impact of the Hospital Indoor Public Spaces on Patients' Health, Assessment, and Analysis
}

\author{
Zhe Du, ${ }^{1,2}$ Baogang Lin ${ }^{1},{ }^{1,2}$ and Zhenyi Chen ${ }^{2}$ \\ ${ }^{1}$ School of Architecture, Xi'an University of Architecture and Technology, Xi'an, Shannxi 710055, China \\ ${ }^{2}$ School of Arts, Xi'an University of Architecture and Technology, Xi'an, Shannxi 710055, China \\ Correspondence should be addressed to Baogang Lin; linbaogang@xauat.edu.cn
}

Received 11 October 2021; Revised 10 December 2021; Accepted 16 December 2021; Published 7 January 2022

Academic Editor: Rahman Ali

Copyright (C) 2022 Zhe Du et al. This is an open access article distributed under the Creative Commons Attribution License, which permits unrestricted use, distribution, and reproduction in any medium, provided the original work is properly cited.

Due to the rapid development of social economy, general hospital buildings of China are undergoing changes in their models, technology, and medical systems. Changes in the model of hospitals may affect the functional structure, streamline layout, and spatial form of the hospital building. The biological-psychological-social (biopsychosocial) model covers the overall significant factors in healthcare. The model focuses on the hospital public environment. For the advocacy of patient-centered medical concepts, researchers have put forward newer and higher requirements as well. However, there is scarcity of research that targets the psychological impact of general hospital public space environment construction on the patients' health. Moreover, there is no unanimously recognized standard questionnaire for the public space environment. The purpose of this article is to investigate the needs of inpatients for the space environment, to understand the influencing factors of various space environments, and to explore the law of patient needs. The research intends to provide a theoretical background for the construction of a patient-centered space environment. A dedicated questionnaire was designed to systematically collect the significant features and factors. Using cluster sampling, a total of four medical and surgical wards were investigated. The data obtained from 430 questionnaires were statistically analyzed by SPSS-10.0. Various statistical operations such as descriptive analysis, independent sample $t$ test, one-way analysis of variance, and linear and logistic regressions were performed over the data. The psychological impact was studied from four aspects. The highest score (3-4 points) obtained for the larger number of patients $(84.4 \%)$ testifies that the public space environment has a significant impact on patients' mental health. Besides healthcare, outcomes of the paper may be used in various related domains such as psychological well beings, spatial analysis, social interaction, and public space designing.

\section{Introduction}

Evaluation of the impact of the hospital indoor public space is important for the well-being of inpatients. However, until recently, the designing of hospitals lacks such healthy considerations. From the 1950s to the present, the development of hospitals in China is roughly divided into three stages. The first stage was from the 1950s to the 1970s, when the country's productivity was relatively backward and the people's living standards were low. Even in this day and age of technology, proper healthcare facilities and the availability of hospital buildings are the challenges [1] to be overcome. Recently, a universal hospital building model is suggested to address this important need [2]. Due to the economic and technological requirements, public space is only to satisfy the people flow, whereas no consideration has been paid to take into account the psychological needs of users. The second stage is from the 1970s to the 1990s. In the said period, considerable advancement and reforms were made and productivity was improved. With the economic boost, the people's living standards were improved. The scale and complexity of hospitals were greatly increased. Hospitals have been equipped with advanced and expensive tools. Emphasis was paid on the teaching function of hospitals, and the importance of logistics was also been recognized. As a result, the three-level functional structure of hospitals was replaced by the five-level functional structure leading to the design of a large-scale building complex [3]. The third stage 
of hospital development is ongoing since the 1990s. With the rapid development of urbanization in the country, the demand for the construction of modern hospitals also increased. There have been many new constructions and expansion projects going on. This brought new development opportunities to the hospital's architectural design [4]. According to the healthcare study reports, medical activities need to be patient centered rather than disease centered. A patient needs to be treated as a person rather than a damaged machine. Self-esteem and care of patients play important roles in the entire diagnosis and treatment process. A whole medical process revolves around the conscious satisfaction of the patients. The goal of a hospital designer is to ensure whether the hospital space truly creates a healthy medical environment. A designer should consider proper spacing for the patient's family members, visitors, and medical staff while designing models of a conducive hospital. Public space refers to the space for patients, family members, medical staff, and other users in the hospital while seeking medical treatment. It is also an important space for taking rest and stress relief. The quality of the space has an important impact on the overall image of the hospital, the psychological thoughts of patients, and emotions of medical staff. People are also becoming aware of the fact that good public space in hospitals plays an important role in promoting the recovery of patients and reducing their suffering. For hospitals, the first group of people to be concerned should be patients, followed by visiting attendants and medical staff. Among them, patients are divided into outpatients and inpatients. From the perspective of the age structure, children and the elderly are more physically and mentally capable. Weak and aged patients require special care, while women and disabled are the vulnerable groups and require special attention. In the era of the global market economy, hospitals have been transformed from the former social welfare and nonprofit unit into a profitable and welfare department. The wellfunctioned [5], comfortable, convenient, friendly, and coordinated public space environment will undoubtedly create a good impression by users. This will also improve the image of the entire hospital and greatly increase the hospital's affinity and cohesion. The hospital has become the center of the community, appearing as a brand-new management agency for physical and mental health. Therefore, meeting the behavioral and psychological needs of users, paying attention to the communication between people, and the communication between people and the environment have become the inevitable requirements for the further development of general hospitals. This forces architects to reflect and explore how to create a modern and humanized public activity space that meets the fundamental needs of people. This is the purpose and significance of the author's choice of this topic for research and discussion.

\section{Related Work}

In recent years, the promotion of "golden health card" and the establishment of a tertiary medical network have gradually replaced the single outpatient registration method by means of online appointment registration [6], electronic registration, and electronic accounting. With the "one card" registration, price adjustment and payment have become easier. By dint of the technology, the patient's frequent visits and traveling has been reduced to a greater extent. The pressure during the peak period of medical visits in various public spaces has been relieved. Personnel has been rationally diverted, and the behavior patterns of patients in public spaces have gradually been optimized. The emergence of the remote medical network has shortened the time and space distance between the family and the hospital. For example, Harbin No. 1 Hospital delivers treatment plans through international computer networks to save patients' lives. The 'ECG phone monitor" developed by the 54th Hospital of Chengdu Military Region can enable patients from thousands of miles away to receive hospital diagnoses [7]. All these factors affect the form, content, and use area of public spaces such as registration, waiting for doctors, and waiting for medicine. At the same time, the above-mentioned technologies increase the proportion of nonfunctional spaces in public spaces.

The development of science and technology has greatly expanded the functions of hospitals. Like the content of medical services, medical buildings have become more complex. But at the same time, the overall structure of the hospital has become more hazy and difficult to understand. The elderly public spaces have become less adaptable to such changes. Under the pressure of huge institutions, it is difficult to effectively link public spaces with different functional departments like hospitals. With frequent rebuilt and expansion of hospitals to accommodate the development of medical technology, public spaces in most of the hospitals have been shrunk. Thus, hospitals have become more chaotic and irregular. Dizzy has become synonymous to public spaces in hospitals. In order to enable patients to have a concise medical process, walking distance for patients is reduced. At the same time, to make a possible relationship between outpatients, hospitalization, medical technology, and other important departments more compact and convenient, some standard hospitals have been erected. Spacious hospitals consist of streets, a shared atrium, and integrated halls for divisions. This improves the sense of spatial direction and the integrity of the entire hospital building space [8].

When, where, and how people's activities and behaviors change are the most basic information in architectural creation. Through the research on environmental psychology in the hospital building, we can truly understand the needs of patients and medical staff to summarize the basic characteristics of the user's behavior. This will provide a base for scientific and rational architectural design. Environmental psychology research must be an important factor in architectural design, paving the way for more humane architectural design [9]. When one is familiar with the environmental psychology of space, people's living space and the relationship between psychological distance and actual distance can be well considered. Similarly, the intersection of personal space and other people's space, and the sense of openness and closure of space be better reflected. During architectural or environmental designing, 
one can organize space appropriately, arrange buildings or building groups carefully, adjust their area, height, and distance reasonably. A design processed in this way not only has the academic basis of environmental psychology but also broadens the design horizon. Thus, the designing process meets the perspective of environmental psychology and the people's psychological requirements. While designating the layout of a hospital building, space should be as spacious and extended as possible. There ought to be smooth lines, a reasonable structure, distinct levels, and an elegant appearance. There should be a variety of design and construction methods such as glass grids, greening of the central hall, flower placement, and decorative embellishments to create a three-dimensional and beautiful environment. Space has to be considered in the designing so as to provide patients and medical staff with a pleasing and easy-to-see work environment for medical treatment. The buildings and facilities of the hospital should be patient centered in every aspect covering the pathological, physical, and psychological requirements of the patients. Moreover, the privacy and convenience of the patients should be considered. The research results of aesthetics and behavioral psychology are to be considered in the designing of the indoor environment to avoid a boring and/or cold indoor space environment. The color, decoration, materials, lighting, sound, audiovisual, and other aspects are to be carefully arranged to create a warm and pleasant atmosphere to relieve the nervousness of the patients [10]. If possible, the outdoor landscape and the indoor environment should be infiltrated and merged with each other as much as possible. In addition, the layout of furnishings, furniture, daily necessities, lamps, and potted plants need to be ensured. It should also be considered that how better the effects of light and shadow are obtained. The indoor atmosphere should be designed in accordance with the psychological laws of the visual environment. All in all, the design of hospital building space should be based on the research studies and opinions of patients and medical staff. Research works have been carried out in the literature to ensure comfortable spatial solutions in hospitals and healthcare buildings. For instance, Lacanna et al. [11] proposed the indoor public space measurement (IPSM) method to facilitate designers and researchers in evaluating patient-centered spatial solutions. The work of Singha [12] mentioned the risks of hospital buildings being constructed without keeping in view the demands and facilitation of users. Similarly, Berdejo-Espinola et al. [13] necessitate the need for public spaces and green spaces for health and healing.

It is convenient for the needs of different people to form a humanized design concept and to create a comfortable, cordial, and detailed working space for medical treatment [14-18]. Therefore, it is the demand of the day to meet the behavioral and psychological needs of users to pay attention to the communication between people and the environment. Keeping in view the facts, architects should strive to create a modern and humanized public activity space while designing general hospitals[19]. The focus should be to meet the fundamental needs of people.

\section{Method}

This research work intends to analyze and assess the psychological impact of public space in healthcare buildings. The data mining techniques are utilized after systematic data elicitation. Through orderly investigation, data were gathered about the current situation and development trend of public space, the function, space, behavior patterns, and psychological needs of users. Details of the research work are presented in the following sections.

3.1. Sampling Method. Cluster sampling was used to sample four internal medicine and surgical wards in a tertiary A hospital. The patients chosen for questioning were suffering from common and frequently-occurring diseases. Because patients in obstetrics, gynecology, pediatrics, emergency, burns, and other departments have their particularities, only relevant questions were asked. Inappropriate facts and findings are not included in this study. The designed questionnaire consists of 3 parts, namely, the basic information of the patient, and the content of the patient's needs and satisfaction. Basic information includes department, age, gender, marital status, ethnicity, occupation, degree of socialization, degree of self-care, agree to participate in medical insurance, family income per capita, number of days in the hospital, days after surgery, number of hospitalizations, whether or not they have lived in other hospitals, and questions about the availability of 14 items in the hospitals. There are a total of 32 requirements. To systematically analyze the impact of the hospital's public space environment on the patient's psychology, the Likert 4-level scoring method is used. For a question, "No" is treated as a 1-point score. In the available literature, there is no standard questionnaire. Therefore, a dedicated questionnaire was devised for this purpose. However, the purpose of the research and suggested standards [8-17] were followed as guided by Maslow's "hierarchy of needs." The hierarchy of needs theory is a theory of the structure of human needs initiated by the American psychologist Maslow [20]. The content mainly involves four aspects ( $\mathrm{C} 1$ to $\mathrm{C} 4)$. $\mathrm{C} 1$ : adequate rest facilities (such as pavilions, balconies, vending machines, and seats) are set up in places where all patients may stay (such as waiting rooms, atriums, corridors, and telephone booths). Thus, the $\mathrm{C} 1$ aspect improves patients a sense of belonging. C2: the ward is quiet and clean with fresh air, which is more conducive to treatment. C3: a good environment in the public space of the hospital, which can avoid people around when waiting for treatment, medicine, or rest, and entertainment in the public space (including patients and medical staff) causing interference to patients. C4: an artistic atmosphere is introduced (such as placing sculptures, paintings, rich spatial colors, and medical treatment accompanied by light music). This aspect can make patients feel happier.

3.2. Survey Object and Method. The eligibility criteria of the survey consisted of patients sober minded, patients over 18 years old, and patients willing to cooperate in the study. 
Other criteria considered were the willingness of the participants to fill in the questionnaire on their own allow participants to fill the questionnaire in case of illiterate individuals. Considering that the patient was not yet familiar with the environment and staff when they were first admitted to the hospital, patients who were hospitalized for more than 3 days were selected for the investigation. The exclusion criteria of the survey ignore patients who were unconscious, mentally abnormal, or who refused to investigate. Using the on-site surveys, investigators intensively distributed the questionnaires in two weeks. The patients, who were unable to answer the question on the spot, were allowed to fill in at their own ease. The questionnaires were then collected after proper filling. Each questionnaire took 10 to 30 minutes. The illiterate individuals' questionnaire can be filled out by the nurse one by one and/or according to their consent. The entire practice was about the subjective feelings of the respondents, and the investigators were not involved in any guiding efforts other than in the case of illiterates. A total of 460 questionnaires were issued, and 452 were retrieved. 8 cases did not complete the survey. The recovery rate was $98.3 \%$. Out of the 452 cases, 22 cases did not meet the requirements, accounting for $4.8 \%$. The excluded cases were being under 18 years of age (4 cases). Moreover, there were missing items in 6 cases. Finally, 430 white-effect questionnaires were identified and considered for onward processing.

\subsection{Evaluation Scale Performance and Data Processing.} The performance of the scale was evaluated from the 430 questionnaires collected. The reliability coefficient was Cronbach's $\alpha$ coefficient, and the correlation analysis (Pearson coefficient) was used to evaluate the validity of the content on the basis of the analysis and evaluation validity standards [21]. The total Cronbach's $\alpha$ coefficient of the four items is 0.9602 , which is larger than 0.9036 in the preliminary survey. The figure indicates that the scale has high internal consistency. The Cronbach's $\alpha$ coefficient in each factor is greater than 0.7 (see Table 1).

All data were fed to SPSS-10.0 for statistical analysis. Descriptive analysis of all requirements was analyzed using the descriptive function. The independent sample $t$ test and one-way analysis of variance (ANOVA) were used to analyze demographic data. The correlation coefficients of related variables were computed, and multiple linear regression analysis and logistic regression analysis were performed. The logistic regression model used is given in the equation as follows:

$$
\ln \left(\frac{P}{Q}\right)=\ln \left(\frac{P}{1-P}\right)=\beta_{0}+\beta_{1} x_{1}+\beta_{2} x_{2}+\cdots+\beta_{m} x_{m}
$$

In statistics, $\ln (P / Q)$ is called logit transformation or logarithmic transformation of $\mathrm{P}$, namely, Logit $\mathrm{P}$.The regression equation thus obtained is called the logistic regression equation, given as in following equation:
TABLE 1: Cronbach's $\alpha$ coefficient.

\begin{tabular}{lcccc}
\hline Factor & C1 & C2 & C3 & C4 \\
\hline Cronbach's $\alpha$ coefficient & 0.9050 & 0.8947 & $\mathbf{0 . 9 2 5 6}$ & 0.8974 \\
\hline
\end{tabular}

$$
P=\frac{e^{\beta_{0}+\beta_{1} x_{1}+\beta_{2} x_{2}+\cdots+\beta_{m} x_{m}}}{1+e^{\beta_{0}+\beta_{1} x_{1}+\beta_{2} x_{2}+\cdots+\beta_{m} x_{m}}} .
$$

Logistic regression model estimated from the sample is given in the equation as follows:

$$
\ln \left(\frac{P}{1-P}\right)=\beta_{0}+\beta_{1} x_{1}+\beta_{2} x_{2}+\cdots+\beta_{m} x_{m} .
$$

\section{Experiments and Discussions}

Besides the proper data collection and analysis, the reliability and validity of the questionnaire were tested. Experimentation was carried in a systematic way on the basis of data obtained from 430 valid questionnaires. Details about sampling are presented in the following subsection.

4.1. Sample General Situation. Within the specified investigation time, there were 472 eligible patients, 12 patients refused the investigation, and 460 patients accepted the investigation. The response rate was $97.4 \%$, which met the specified requirements. A total of 460 questionnaires were issued while 452 were recovered; 8 of which were not completed; thus, the recovery rate was $98.3 \%$. Out of the 452 questionnaires, 22 did not meet the requirements; and finally, 430 qualified questionnaires were considered. Among 430 patients, 271 were males, accounting for $63 \%$, and 159 were females, accounting for $37 \%$. The youngest is 18 years old, and the oldest is 85 years old, with an average range of $49.13 \pm 16.54$ years old. There were 216 medical patients and 214 surgical patients included in the survey. At the time of the investigation, the number of hospitalization days was the lowest of 3 days, and the longest was 270 days, with an average of $13.57 \pm 20.49$ days. The total average value of nursing needs is $3.39+0.41$ points, the lowest score is 2.09 points, the highest is 4.00 points, and the $95 \%$ confidence interval is $2.60-4.0$ points. There were 67 persons with a score of less than 3.0, accounting for $15.6 \%$, and 363 persons with a score of 3.0 to 4.0 , accounting for $84.4 \%$. 57 cases scored a full score of 4.0 , accounting for $13.3 \%$.

4.2. Analysis of the Psychological Influence of Patients in Various Aspects. All space environment projects are composed of 4 aspects: C1, C2, C3, and C4.Among the 4 aspects, the highest average value is "C 3 " (3.4843 points), and the lowest is "C4" (3.2378 points) (see Table 2).

Comparing the psychological impact of hospital public space environment on medical and surgical patients, we found no significant difference in the average scores of the departments. Moreover, there is no significant difference in the needs of medical and surgical departments in terms of the four aspects (see Figure 1). 
TABle 2: Comparison of 4 aspects.

\begin{tabular}{lccr}
\hline 4 aspects & $\overline{\mathbf{x}}$ & $\overline{\mathbf{x}} \pm$ SD & 95\% CI \\
\hline C1 & 3.37 & $3.37 \pm 0.49$ & $2.20 \sim 4.00$ \\
C2 & 3.37 & $3.37 \pm 0.47$ & $2.32 \sim 4.00$ \\
C3 & $\mathbf{3 . 4 8}$ & $3.48 \pm 0.44$ & $2.57 \sim 4.00$ \\
C4 & 3.24 & $3.24 \pm 0.58$ & $2.19 \sim 4.00$ \\
\hline
\end{tabular}

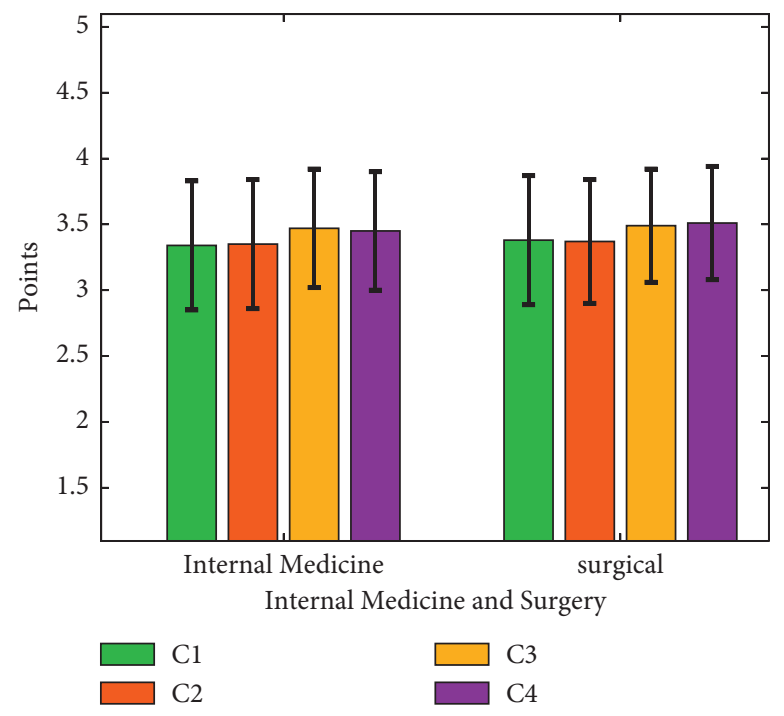

Figure 1: Comparison of psychological effects of medical and surgical patients.

4.3. Analysis between Different Ages. With reference to the World Health Organization (WHO) classification of age, 18-44 years old are classified as a youth group, 45-59 years old as the middle-aged group, 60-74 years old as the elderly group, and older than 75 years old as the old people group. Taking into account the fact that young people under 44 years old will be very different around the age of 30 , it was considered as a dividing line. Thus, the 18-30 years old was treated as the youth group and the 31-44 years old as the middle-aged group. For seamless analysis, participants were categorized into five groups. Differences in perception were observed between $\mathrm{C} 1$ and C2 in different age groups. Therefore, the LSD (least significant difference) method was used for pairwise comparisons [22]. The results are shown in Figures 2 and 3, showing that young people feel lower about $\mathrm{C} 1$ than those in middle-aged and old people. Middle-aged, young, and old people have higher perceptions of $\mathrm{C} 2$ than young people.

4.4. Differences between Different Occupations. Patients of different occupations have different perceptions about the C3 aspect. Therefore, the LSD (least significant difference) method is used for pairwise comparisons (see Figure 4). It shows that the physiological needs of the farmers are higher than other workers. For the rest of the groups, there was no obvious difference.

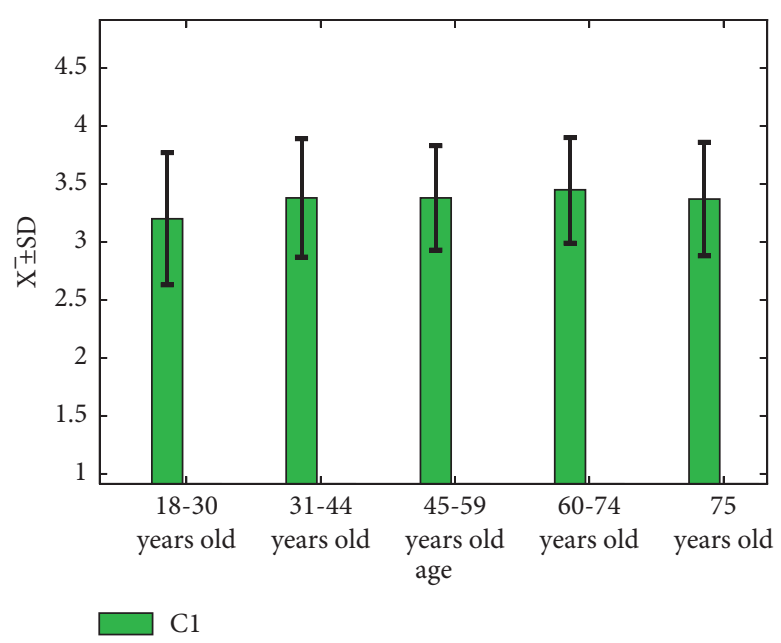

Figure 2: Differences of $\mathrm{C} 1$ in different age groups.

There is a difference in the demand for C4 among the same occupational patients, as shown in Figure 5. The demand of farmers is higher than that of the worker group and the cadre group. For the rest of the groups, no significant difference was observed. 


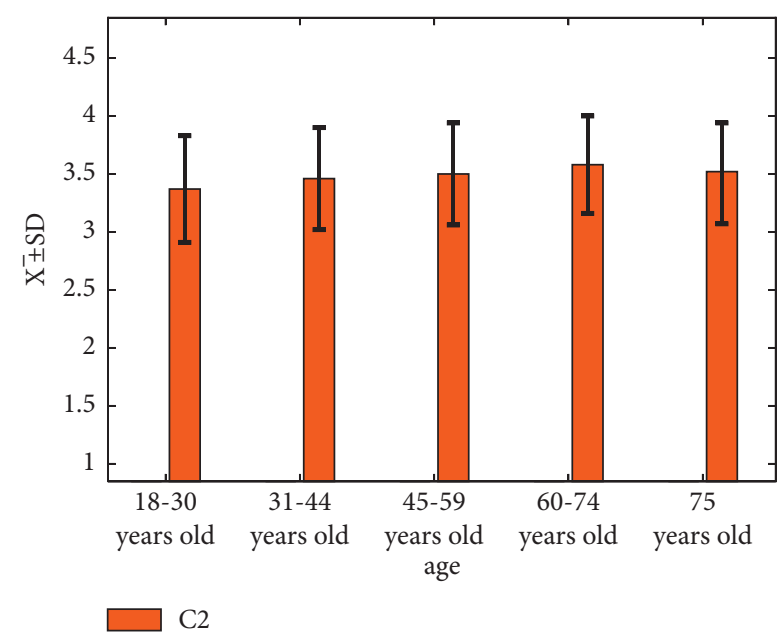

Figure 3: Differences of C2 in different age groups.

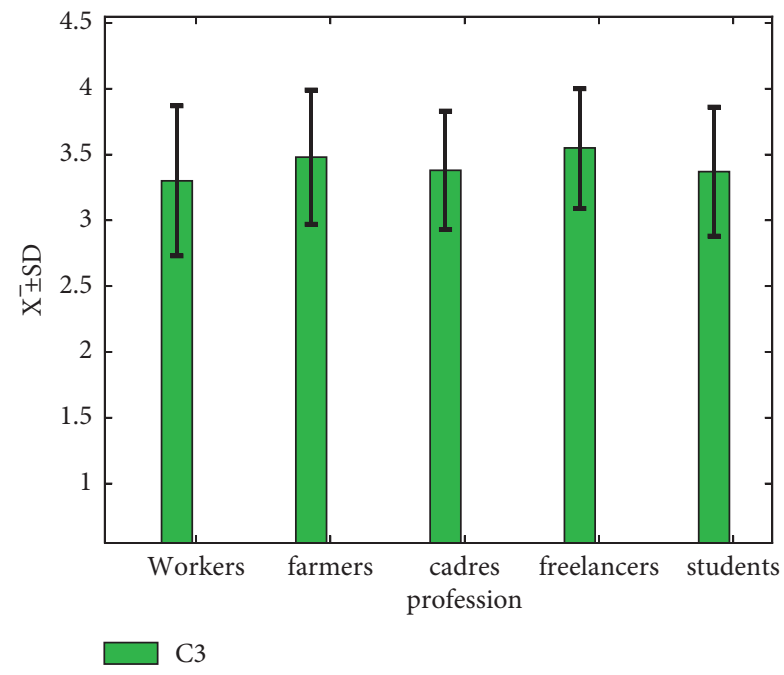

Figure 4: Differences of C3 in different occupations.

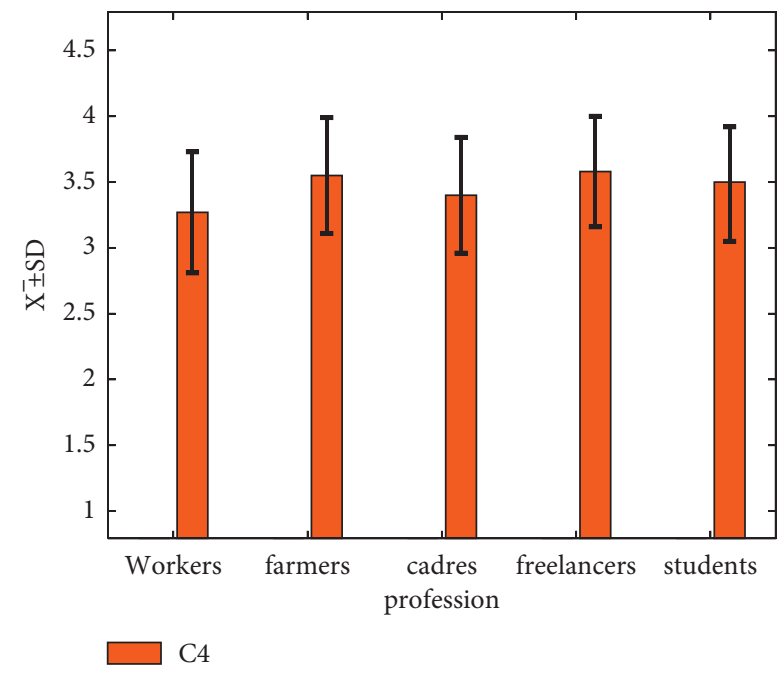

FIGURE 5: Differences of C4 in different occupations. 


\section{Conclusion}

A hospital building is a magical place for human beings to survive, recover, maintain health, and resist diseases [23]. Hospitals are the distinct and distinguished buildings deemed to offer all the healthcare facilities required in medical activities. As a special type of civil building, it has extremely complex functions and is constantly developing and changing. Being an important part of the hospital, public space refers to the space where patients spend the longest time during their stay in a hospital. Such spaces are frequently visited by patients during their treatment and/or during rest. These are the places that contribute to making the first impression. It is why the design of the public space environment typically has an important impact on the patient's treatment and rehabilitation process [24]. It has been suggested [25] that the depression and fear of patients may be combated by the feeling of being at home. Architects should plan to depict the identity of a home so that during treatment and hospitalization a patient perceives a homelike environment. This is the aim of the agreed-upon biological-psychological-social-medical model [25]. In the dedicated investigation, it was found that different patients feel differently about the public space in the medical building. The functions of hospitals are extremely complicated and are often interspersed with various streams. In such situations, patients suffering from illness and medical workers with high workloads suffer badly. As per the outcomes of psychological research, it is suggested that advanced techniques of architectural design should be followed to understand the users' need and to get twice the result with half the effort. This research work is based on the impact of environment. Using a systematic questionnaire, data were gathered from a large number of patients. An endeavor is made to suggest a framework for the state-of-the-art designing of hospitals so as to satisfy all the psychological needs of users. Though some hospitals are systematically designed and have enough public space [26], little consideration is paid towards capacity and space during the designing of hospitals. This article discusses the issue in a detailed way and suggests a conducive and patient-centered design structure for the hospital buildings. As a future strategy, we are determined to enhance the model by making use of machine learning classifiers. The model will automatically suggest patient-centered parameters based on the trained dataset and real-world data.

\section{Data Availability}

The datasets used and analyzed during the current study are available from the corresponding author upon reasonable request.

\section{Conflicts of Interest}

The authors declare that they have no conflicts of interest.

\section{References}

[1] P. Lamarche, R. Pineault, J. Gauthier, M. Hamel, and J. Haggerty, "Availability of healthcare resources, positive ratings of the care experience and extent of service use: an unexpected relationship," Healthcare Policy | Politiques de Santé, vol. 6, no. 3, pp. 46-54, 2011 Feb.

[2] Y. Nagasawa, "Global Hospitals in 2050-A review of the historical development of hospital building studies from a global perspective," Japan Architectural Review, vol. 3, no. 1, pp. 5-24, 2020 Jan.

[3] M. Aoki, M. Maruoka, Y. Ikuta, and N. Sahasi, "Case history of raft foundation behavior for a large scaled building complex," in Proceedings of the Third International Conference Case Histories in Geotechnical Engineering, St Louis, MI, USA, June 1993.

[4] J. A. Dvoskin, S. J. Radomski, C. Bennett et al., "Architectural design of a secure forensic state psychiatric hospital," Behavioral Sciences \& the Law, vol. 20, no. 5, pp. 481-493, 2010.

[5] J. Hashimoto, "How had the wartime planned economic system distorted the well-functioned management system of Japanese large company: a case study of N corporation," Hosei Journal of Business, vol. 38, 2001.

[6] X. H. Zhao, "Making process of golden heart source health card and soft heart source health plaster," Journal of Health, vol. 16, no. 12, pp. 200-211, 2005.

[7] L. J. Mena, V. G. Félix, A. Ochoa et al., "Mobile personal health monitoring for automated classification of electrocardiogram signals in elderly," Computational and mathematical methods in medicine, vol. 29, 2018.

[8] M. Zhang and X. L. Fu, "A discussion about the Influence of the development of medical pattern on hospital building form," Architectaral Journal, 2002.

[9] A. Ernst and U. Wenzel, "Bringing environmental psychology into action," European Psychologist, vol. 19, no. 2, pp. 118-126, 2014.

[10] K. J. Ritchey, "Panoramic image based virtual reality/telepresence audio-visual system and method," Information System, vol. 22, no. 12, pp. 12-24, 1996.

[11] G. Lacanna, C. Wagenaar, T. Avermaete, and V. Swami, "Evaluating the psychosocial impact of indoor public spaces in complex healthcare settings," HERD: Health Environments Research \& Design Journal, vol. 12, no. 3, pp. 11-30, 2019 Jul.

[12] S. Singha, Future Healthcare Design, Routledge, London, UK, 2020.

[13] V. Berdejo-Espinola, A. F. Suárez-Castro, T. Amano, K. S. Fielding, R. R. Oh, and R. A. Fuller, "Urban green space use during a time of stress: a case study during the COVID-19 pandemic in Brisbane, Australia," People and Nature, vol. 3, 2021.

[14] Y. F. Zhang, Y. Zhou, and W. J. Li, "Study on reorganization of patients and nurses on nursing service Needs," Modern nurse, vol. 11, no. 24, pp. 2074-2076, 2005.

[15] X. J. Jiang, "Investigation and analysis of the needs of 120 inpatients," Journal of North Sichuan Medical College, vol. 12, no. 1, pp. 83-84, 1997.

[16] W. L. Shao, X. Yang, and S. J. Li, "An assessment of inpatients needs and satisfactions to nursing care and related factors," Chinese Journal of Nursing, vol. 40, no. 12, pp. 888-890, 2005. 
[17] X. X. Zheng and Y. Zhao, "Investigation and analysis of the nursing needs of 263 inpatients," Chinese Journal of Practical Nursing, vol. 19, no. 5, pp. 51-52, 2003.

[18] Y. M. Guan and Q. Zhang, "Investigation and analysis of the needs of inpatients in the second-level hospitals in the special zone," Chinese Nursing Management, vol. 14, no. 2, pp. 46-48, 2004.

[19] J. Wang, Y. L. Liu, X. Wang, W. Y. Wan, M. Y. Zhao, and L. H. Wu, "Qualitative research on inpatients' nursing needs," Journal of Modern Nursing, vol. 30, pp. 160-162, 2006.

[20] J. O. Prochaska, C. C. Diclemente, and J. C. Norcross, "Search of how people change: applications to addictive behaviors," American Psychologist, vol. 47, 1992.

[21] J. E. Helms, K. T. Henze, T. L. Sass, and V. A. Mifsud, "Treating cronbach's alpha reliability coefficients as data in counseling research," The Counseling Psychologist, vol. 34, no. 5, pp. 630-660, 2006.

[22] X. Lu, Y. Xi, and D. Lu, Stock Dividend Is the Natural Choice of Strong Companies: Based on Least Significant Difference Method, Springer books, Berlin, German, 2005.

[23] L. Y. Thi and H. E. Muller, "Ingested Listeria monocytogenes survive and multiply in protozoa," Journal of Medical Microbiology, vol. 33, 1990.

[24] E. Wressle, A.-M. Eeg-Olofsson, J. Marcusson, and C. Henriksson, "Improved client participation in the rehabilitation process using a client-centred goal formulation structure," Journal of Rehabilitation Medicine, vol. 34, no. 1, pp. 5-11, 2002.

[25] K. Halbritter, J. Beyer-Westendorf, J. Nowotny, S. Pannach, E. Kuhlisch, and S. M. Schellong, "Hospitalization for vitamin-K-antagonist-related bleeding: treatment patterns and outcome," Journal of Thrombosis and Haemostasis, vol. 11, no. 4, pp. 651-659, 2013.

[26] J. Tang, The Necessity of Optimal Described the Design of Public Space in Residential Construction, Fujian Architecture \& Construction, Fujian, China, 2005. 